zudem relativ viele Jugendliche, die sehr häufig Cannabis konsumieren. Über 5\% der 15-Jährigen berichten hier über einen Cannabiskonsum von 40 mal oder mehr im letzten Jahr (Dauerkonsum). In den meisten Ländern ist die Rate der Dauerkonsumenten jedoch eher klein. In Deutschland zählen etwa 3\% der 15-Jährigen zu den Dauerkonsumenten, weitere $8 \%$ berichten über einen Freizeitkonsum (3 bis 39 mal im letzten Jahr).

\section{Fazit}

Die hier berichteten Ergebnisse weisen eindrucksvoll darauf hin, dass Erfahrungen mit Tabak, Alkohol und Cannabis - selbst im Alter von 11 bis 15 Jahren - in allen an der Studie teilnehmenden Ländern üblich sind. Auch wenn sich diese Erfahrungen vielfach auf einen Experimentier- oder Probierkonsum beschränken, wie er im Jugendalter als "normal« bezeichnet werden kann, entwickelt ein beträchtliche Zahl an Jugendlichen daraus einen regelmäßigen Konsum. So gibt es in Deutschland bei den 11- bis 15-Jährigen bereits vor Erreichen des gesetzlich vorgeschriebenen Mindestalters für Tabak- und Alkoholkonsum eine Teilgruppe von Jugendlichen, die regelmäßig Alkohol trinkt (13\%) und/oder Tabak raucht (15\%). Hinzu kommen etwa $11 \%$ der Jugendlichen, die als Freizeit- und Dauerkonsumenten von Cannabis gelten müssen. Die Ergebnisse der Studie machen deutlich, dass hier bisher weder die entsprechende Jugendschutzgesetzgebung noch einschlägige Kampagnen und Präventionsstrategien eine durchschlagende Wirkung erzielt haben. Gemessen an der gemeinsamen Zielstellung der Weltgesundheitsorganisation, das Rauchen bei jungen Menschen im ersten Jahrzehnt des neuen Millenniums auf ein Mindestmaß zu reduzieren, ist die Bundesrepublik Deutschland zudem Schlusslicht im internationalen Vergleich. Wie die dargestellten Ergebnisse ebenfalls zeigen, nimmt der regelmäßige Konsum psychoaktiver Substanzen um das 13. Lebensjahr zu. Aus diesem Grund ist es erforderlich, spätestens in der 5. Jahrgangsstufe mit präventiven Maßnahmen zu beginnen. Nur so kann der Beginn regelmäßigen gesundheitsschädigenden Verhaltens - wenn er sich nicht ganz vermeiden lässt - auf einen späteren Zeitpunkt verlagert werden.

\section{Matthias Richter, Dipl.-Soz.}

Universität Bielefeld - School of Public Health

HBSC $\mid$ Health Behaviour in School-aged Children Postfach 1001 31; 33501 Bielefeld/Germany fon: 0521.106-3878 fax: 0521.106-6433 email:matthias.richter@uni-bielefeld.de

\section{Fußnote:}

1 Currie, C., Roberts, C., Morgan, A., Smith, R., Settertobulte, W., Samdal, O., Barnekow Rasmussen, V. (eds.): Young people's health in context - Health Behaviour in School-aged Children (HBSC) study: International report from the 2001/02 survey. (Health Policy for Children and Adolescents, No. 4) Kopenhagen: WHO-Europe

STANDPUNKT

\title{
Im Parallelluniversumvon Köln-Mülheim
}

\author{
Elmar J. Koenen
}

Wie der Staat sich von seinen Gegnern vorführen lässt (SPIEGEL). So oder ähnlich tönte es politisch quadrophon von allen Medienseiten, nachdem die Polizei vor ein paar Wochen in einer Wohnanlage in Köln-Chorweiler statt des Mieters Metin Kaplan, des >Kalifen von Köln< nur einen Zettel an seiner Wohnungstür vorgefunden hatte. Der informierte darüber, dass der Schlüssel beim Nachbar sei (und es daher eigentlich unnötig sei, die Tür aufzubrechen). Von oben Mitte (Silke Stokar, GRÜNE) bis rechts unten (Rüttgers/Beckstein, CDU/CSU), von vorne an der Wand (Westerwelle, FDP) bis hinten Mittelinks (Sonntag-Wollgast, SPD) die nämliche, dämliche Aufregung über eine angeblich blamable >Vorführung` des Rechtsstaats.

Vorgeführt wurde - im ganz anderen, unmetaphorischen Sinn des Wortes - in der Tat die Kompliziertheit unseres Rechtsstaats, dessen Verfahren lange überlegt und bewährt sind, auch stets weiterentwickelt werden und dabei nur noch komplexer geworden sind und dessen Mechanismen nur Spezialisten wirklich verstehen. Nichts Neues auch die Einsicht, dass jene Verfahren immer wieder auch zu politisch unerwünschten Resultaten führen.

Die erstaunlich schlichte, oft zitierte Klage von Altbundeskanzler Schmidt, dass er seine Wasserrechnung nicht mehr nachvollziehen könne, war kein Grund für eine entsprechende Verwaltungsreform, und es macht auch keinen Sinn, wenn Friedrich Merz seine Vorschläge zur Steuerreform damit begründet, dass eine Steuererklärung dann auf einen Bierdeckel passe. Kaum einer der Millionen, die einen PC bedienen ode Auto fahren, haben je das Funktionieren ihre Lieblingsspielzeuge verstanden. In der Tat reich es auch, dass die Benutzeroberflächen einfach und übersichtlich strukturiert sind und den Benutzer nicht mit genaueren Kenntnissen des Funktionierens belasten. Bei der Mobilisierung von Recht bedeutet das, dass wir uns einen Anwalt unseres Vertrauens suchen, den wir dafü bezahlen, dass er uns mit der rechtlichen Komplexität unserer Fälle und Unfälle nicht behelligt.

Aber noch einmal zurück zu den Räuber-Gendarm-Interaktionen in Köln-Mülheim, KölnChorweiler und Köln-Nippes. Dort liegen an de Neusser Strasse und am Niehler Kirchweg die beiden Zentren des inzwischen verbotenen Kalifats, zwei mit Sichtblenden und Stahltoren für Ungläubige unzugängliche und uneinsehbare Grund- stücke. Der eigene eigene Fernsehsender >Hakk-TV die Verbandszeitschrift ,Gemeinde Mohammeds und eine eigene Verfassung mit 15 ungläubigenfeindlichen Artikeln, machten dieses Reich zum Kleinstaat im Staat. Das waren die Spielorte einer politischen Wanderbühne, auf der nichts weniger als die Organisierung der islamischen Weltherrschaft geprobt wurde. Hervorgegangen war die deutsche Dependance ursprünglich aus den internen Machtkämpfen und Fraktionierungen im Prozess der Islamisierung der Türkei, dessen Beginn in die frühen 80er des vergangenen Jahrhunderts fällt (Eine kurze Zusammenfassung findet man im Internet unter http://www.nahost-politik.de/islam/kalifatstaat.htm)

Es war damals sicher keine zufällige Entscheidung von Cemaleddin Kaplan, (dem Vater des inzwischen zur persona non grata gewordenen Metin Kaplan), seinen später bis zu 7000 Muslime umfassenden >Kalifatstaat in Deutschland zu gründen. Auch in der Türkei war natürlich bekannt, dass der funktionierende soziale Rechtsstaats seine nicht beschäftigten Einwohner relativ großzügig alimentiert (Die Stadt Köln fordert gerade von Kaplan junior 150000 Euro erschlichene Sozialhilfe zurück) und Beschuldigte wie Angeklagte mit einem hohen Maß an liberalen $\mathrm{Ab}$ wehrrechten gegen staatliche Zugriffe ausstattet, die z.B. eine Abschiebung von Ausländern immer noch erschweren. Dass mühsam entwickelte soziale und rechtliche Errungenschaften einer Gesellschaft genutzt und missbraucht werden (können), und zwar von Inländern wie von Ausländern, ist keine neue Einsicht. Und schon gar nicht taugt sie zur Begründung der Einschränkung oder Abschaffung von sozialer und rechtlicher Sicherheit. Schwer verständlich bleibt schließlich, wie die öffentlichen Meinungen über ihrer gleichgeschalteten Empörung vergessen können, dass Auseinandersetzungen wie die mit dem militanten Islam v.a. politisch entschieden werden. Der verängstigte Ruf nach weiterer Verschärfung der Gesetze verrät eine soziokulturelle Hilflosigkeit, die dem gegnerischen Politikangebot nichts entgegenzusetzen weiß. In dieser Situation sollte man zumindest auf den guten Gründen unserer gewachsenen Rechts- und Sozialkultur insistieren. 